Beata Grzeszczakowska-Pawlikowska

Universität Łódź, Łódź

DOI: $10.19195 / 0435-5865.142 .13$

\title{
Störungsfaktoren im Bereich der universitären Wissensvermittlung in gesprochener Sprache
}

\section{Zielsetzung}

Der vorliegende Beitrag setzt sich zum Ziel, der Problematik von Kommunikationsstörungen in gesprochener Sprache nachzugehen, die bei der universitären Wissensvermittlung im interkulturellen Kontext auftreten können. Interkulturelle Kommunikation, die auf Grund gewachsener Mobilität von Studierenden weltweit mittlerweile zum Unterrichtsalltag der Hochschulen und Universitären gehört, darf allerdings nicht vereinfachend auf die Verwendung einer bzw. mehrerer Fremdsprachen reduziert werden. Neben den rein (system-)sprachlichen Aspekten gibt es auch weitere Dimensionen, wie etwa die pragmatisch-gesellschaftliche, die historische, die bildungs-traditionelle und nicht zuletzt die individuelle, die für die Prägung der jeweiligen Kultur von Bedeutung sind und die in ihrer Verschränkung den Begriffsinhalt von Interkulturalität als kulturelle Überschneidungssituation füllen (vgl. Wierlacher 2003: 260; auch Straub 2007: 15). Diese Faktoren gelten zugleich, wie hier angenommen wird, als potenzielle Ursachen für Kommunikationsstörungen, zu denen es beim Zusammentreffen verschiedener Kulturen ebenfalls in den Lehr- und Lernprozessen kommen kann. Eine zweckgerichtete Reflexion dieses Problemfeldes ist folglich zur Sicherung effizienter Wissensvermittlung sowie -aneignung unabdingbar. Den ersten Schritt für die Optimierung der jeweiligen Kommunikationsabläufe stellt dabei ein systematisches, empirisch gesichertes Erfassen der jeweiligen Kommunikationsstörung(en) wie auch deren Ursache(n) in konkreten Kommunikationssituationen dar. Im Folgenden soll dies am Beispiel des studentischen Seminarreferats in der Fremdsprache Deutsch als einer interkulturell geprägten Redesituation, in der akademisches Wissen an andere vermittelt wird, erfolgen. Die Beeinträchtigungen der Kommunikation können nämlich insgesamt ungewollten Wirkungen auf der Rezipientenseite zugrunde liegen - im schlimmsten Fall führen sie sogar zum Abbrechen der Kommunikation. 
Der Diskussion ausgewählter Untersuchungsergebnisse geht das Umreißen einiger theoretischer Positionen zur gesprochenen Sprache, zur Rolle der Universität als Wissensvermittler und nicht zuletzt zu Kommunikationsstörungen als Normalität interkulturellen (Sprach-)Handeln voraus.

\section{Gesprochene Sprache}

In dem vorliegenden Beitrag wird von der ganzheitlichen Betrachtung der gesprochenen Sprache ausgegangen. Gemeint ist damit eine simultane Verortung kommunikativer Prozesse in Mündlichkeit auf der verbalen, para- und nonverbalen Ebenen. Diese Auffassung korrespondiert mit dem Konzept somatischer Kommunikation, in dessen Fokus die Körperlichkeit des Sprechens steht (vgl. Scollon / Scollon 1995: 27). Zentral hierbei ist das Kriterium von Mehrkanaligkeit mündlicher Kommunikation, d.h. die gleichzeitige Nutzung akustischer und visueller Zeichensysteme bei der Produktion und Rezeption eines kommunikativen Aktes. Die Gestalt einer mündlichen Äußerung gilt somit als komplexes Gefüge und darf - trotz irritierender Fälle von Nicht-Übereinstimmung zwischen sprachlichen, parasprachlichen und mimisch-gestischen Signalen - keinesfalls auf die verbale Form reduziert werden (vgl. Quasthoff 1996: 9 ff.). Insofern gilt der Körper als „eine vielfältige und komplexe Ressource für kommunikative Erscheinungsformen" (Hausendorf 1997: 406). Neben den paraverbalen Komponenten (Sprechweise / Sprechausdruck) wie auch den nonverbalen Komponenten (Körperausdruck) gehören dazu auch weitere über die taktilen, olfaktorischen, thermalen, gustatorischen etc. Reize übermittelte Kommunikationsanteile (vgl. auch Scherer 1970: 3 f.). All diese Ausdrucksmittel stellen einen festen Bestandteil unterschiedlicher Verhaltensreaktionen der Kommunikationspartner auf die übertragenen Inhalte dar. Ohne zahlreiche außersprachlich vermittelte Informationen wäre die Gesamtheit sozialer Interaktionen schlechthin nicht möglich.

Mit der Auffassung über die Multimodalität von Sprechen wird in dem vorliegenden Aufsatz über den verengten dennoch nach wie vor präsenten Blickwinkel beim Gebrauch der Termini Mündlichkeit / mündliche Kommunikation / gesprochene Sprache, d.h. deren ausschließlicher Identifizierung mit dem akustischen Signal, konsequent hinausgegangen (vgl. Fiehler et al. 2004: 14 f.). Vielmehr rücken hier die komplexen Wechselwirkungen zwischen den verbalen, para- und nonverbalen Kommunikationssignalen in den Mittelpunkt der Erforschung der gesprochenen Sprache. Dabei ist die Ziehung einer Grenze zwischen den einzelnen Kommunikationsebenen - trotz deutlicher Trennungslinie ,entlang der Unterscheidung Stimmgebundenheit (akustische Wahrnehmung) vs. Leibgebundenheit (optische Wahrnehmung)“ (Ebd.: 67) einerseits und Sprache (Inhalt) andererseits - nur schwer möglich. Umso mehr, weil die Erfassung und somit auch die Beurteilung 
der gesprochenen Sprache in der face-to-face-Kommunikation jeweils zugleich unter dem auditiven und visuellen Aspekt abläuft (vgl. auch Potapova 2003: 275).

\section{Die universitäre Wissensvermittlung}

Die traditionelle Rolle der Universität / Hochschule besteht nach dem Humboldtschen Prinzip aus der Einheit von Forschung und Lehre zum einen in der institutionellen Wissensgewinnung, zum anderen in der institutionellen Wissensvermittlung (vgl. z.B. Wiesmann 1999: 28 ff.). Der Universität / Hochschule als Wissensträger kommt vor allem eine doppelte Professionalisierungsfunktion zu, die sich in der wissenschaftlichen sowie der wissenschaftssprachlichen Sozialisation der Studierenden manifestiert und sich somit auf deren Fähigkeit zum sprachlichen Handeln in Schriftlichkeit und Mündlichkeit innerhalb dieser Institution auswirkt (vgl. Guckelsberger 2005: 28). Für die deutschsprachigen Universitäten mit einem grundlegend dynamischen Wissenskonzept sind sog. eristische, diskursive Sprachhandlungsformen, die den wissenschaftlichen Streit konstituieren, besonders kennzeichnend (vgl. z.B. Ehlich 1993: 28 ff.). Die Wissenschaftskommunikation im deutschsprachigen Raum wird dabei in hohem Maße vom mündlichen Diskurs, d.h. von Mündlichkeit und Dialog zugleich geprägt (vgl. auch Venohr 2008: 307). Diesbezüglich spricht Venohr von wissenschaftlichem bzw. wissenschaftsbezogenem Sprechen (vgl. Ebd.: 305). Zum wissenschaftlichen Sprechen an Hochschulen, das ebenso wie das wissenschaftliche Schreiben in standardisierten Formen stattfindet, zählt sie ebenfalls mündliche Prüfungen sowie Sprechstunden. Ferner sind es auch Vorlesungen, Seminare, Seminargespräche, Übungen und Praktika. In solchen diskursiven Kommunikationsformen sollen die Studierenden von ihren Lehrpersonen ,an das systematische Fragen sowie die Debatte“ (Thielmann 2009: 52) herangeführt werden.

Eine besondere Form der universitären Wissensvermittlung, an deren Beispiel - wie einleitend angedeutet - den potenziellen Ursachen für Kommunikationsstörungen in Mündlichkeit auf den Grund gegangen werden soll, stellt das studentische Seminarreferat dar. In dieser Form findet die Vereinbarung von Selbstqualifizierung der Vortragenden (sowohl in fachlicher als auch in wissenschaftsmethodischer Hinsicht) und von Qualifizierung der Mitstudierenden durch die Vortragenden als Wissensvermittler statt (vgl. Guckelsberger 2005: 44 f.). Folglich ist sie für die Umsetzung der o.g. doppelten Professionalisierungsfunktion der Universität besonders gut geeignet. In der einschlägigen Literatur erfährt das studentische Seminarreferat keine eindeutige kategoriale Zuordnung. Es fungiert entweder als Textsorte oder als Diskursart / -typ oder als kommunikative Gattung. Derartige Unsicherheiten resultieren vor allem aus dem Umstand, dass sich in dem studentischen Seminarreferat eine komplexe mediale Verschränkung 
von Schriftlichkeit und Mündlichkeit vollzieht. Diese manifestiert sich in Form gradueller Abstufungen in Abhängigkeit von einzelnen Handlungsschritten bei dessen Produktion - von der schriftlichen Anfertigung über die mündliche Präsentation bis hin zu deren nachträglicher Verschriftlichung in Form einer Seminararbeit (vgl. z.B. Klinger 2009: 13). Angesichts dessen lässt sich das Referat wohl in Anlehnung an Gutenberg (2001: 583) am besten zu den mündlich realisierten schriftkonstituierten Textsorten zuordnen. Auf Grund unterschiedlicher Anteile an geschriebener und gesprochener Sprache stellt das Referat insgesamt eine durchaus multimediale Kommunikationsform dar. Die Multimedialität von Referaten kommt nicht zuletzt in der Verwendung von PowerPoint zum Ausdruck, sodass die übertragenen Wissensbestände zusätzlich in visueller Form mittels Bild und Schrift präsentiert werden (vgl. Ventola 2007: 116).

In dem vorliegenden Beitrag gilt das studentische Seminarreferat als rhetorische Gattung der Rede (als Vortrag). Im Prinzip handelt es sich dabei um eine Informationsrede mit möglichen argumentierenden Passagen. Die Notwendigkeit informierenden Sprechens ist vor allem aus der institutionellen Funktion der Universität, wissenschaftliches Wissen an andere zu vermitteln, abzuleiten. Vor dem Hintergrund der fundamentalen Dialogizität der zwischenmenschlichen Kommunikation ist dabei Rede - trotz deren monologischer Wesensart auf der Performanzebene - als ,,virtuell-dialogische, intendierte Verständigungshandlung eines Redners für meist mehrere Hörer“ (Meyer 2013: 121) aufzufassen. In der kommunikativen Wirklichkeit schlägt sich dies speziell in dem Perspektivenwechsel vom Redeproduzenten zum Adressaten, der bereits in der Redevorbereitungsphase und demnächst beim Vortragen selbst mittels sprecherischer und nonverbaler Ausdrucksmittel vollzogen werden soll, nieder. Mit der Perspektivenübernahme kann die Hörerorientierung des Vortragenden gesichert werden, die - wie hier angenommen wird - einen Beitrag zur Perzeption des im Referat übertragenen Wissens und somit zur störungsfreien Abwicklung der kommunikativen Abläufe leistet. Als rhetorische Kategorie ist Hörerorientierung auf unterschiedlichen Ebenen angesiedelt: auf der Ebene der Sprache (angemessene Verwendung verständlicher Lexik und einfachen Satzbaus), auf der denklogischen Ebene (eine klare von außen erkennbare Strukturierung / Redegliederung) und auf der para- und nonverbalen Ebene (Situationsangemessenheit im Sprech- und Körperausdruck) (vgl. Grzeszczakowska-Pawlikowska 2016: 77 ff.).

\section{Kommunikationsstörungen/-konflikte. Ungewollte kommunikative Wirkungen}

Die verschiedensten Kommunikationsstörungen haben an der kommunikativen Wirklichkeit ihren festen Anteil und sind keineswegs zufällig. Laut Hirschfeld et al. (2010: 44; unter Bezugnahme auf Deppermann / Spranz-Fogasy 2002) ergeben sie 
sich zunächst aus der Tatsache, dass die zeitgleich verlaufenden Gedankenkonfigurationen während des Sprechens linearisiert werden. Dadurch wie auch durch den gedanklich-sprachlichen Rückbezug auf das Vorausgegangene entstehen gewisse „Komplexierungen, [...] die von Anderen nur zu oft als unzulässiger oder verwirrender Reduktionismus wahrgenommen werden" (Hirschfeld et al. Ebd.). Auf Grund von Flüchtigkeit / Kurzlebigkeit der gesprochenen Sprache als konstitutives Merkmal für zahlreiche kommunikative Praktiken (vgl. Fiehler et al. 2004: 52 ff.) entfällt zugleich eine Reihe von Maßnahmen, die das Aufnehmen und Verstehen schriftlicher Texte beträchtlich erleichtern. Gemeint ist damit beispielsweise die Möglichkeit, den geschriebenen Text in eigenem Tempo, bei Bedarf sogar mehrmals, zu lesen oder sich mit visueller Textgestaltung (Texttypographie) zu helfen.

Im Hinblick auf mögliche Kommunikationsstörungen sei zunächst in Anlehnung an Hirschfeld et al. (2010: 44) zwischen form- und kulturbedingten Störungen zu unterscheiden. Zu den ersteren gehören diejenigen Störungen, die allein aus der Form der jeweiligen Äußerungen, sprich: aus dem Sprachsystem resultieren. In der einschlägigen Literatur werden solche systeminhärenten Kommunikationsstörungen als Kommunikationskonflikte bezeichnet (vgl. Ungeheur 1969; zit. nach Hirschfeld et al. Ebd.). Gerade in der sprechsprachlichen Kommunikation manifestieren sich diese laut Ungeheuer (1969: V ff.) in der partiellen Verdeckung oder Verzerrung des übermittelten Inhalts schon auf Grund einer empfindlichen Reaktion des / der Rezipienten auf die Äußerungsform. Im Endeffekt kommt es zu Missdeutungen des Gemeinten, sogar zu Irritationen und Verletzungen, kurzum: zu den bereits erwähnten ungewollten Wirkungen auf der Rezipientenseite (vgl. auch Hirschfeld et al. Ebd.: 45). Im Konfliktbegriff finden dabei - abgesehen von unterschiedlichen Konzeptualisierungen bzw. empirischem Vorgehen - auffallend deutlicher als im Störungsbegriff die Auswirkungen der gestörten Kommunikationsgeschehnisse ihren Ausdruck: Typisch für das Auftreten eines Konflikts sei die Unterbrechung des bisherigen Handlungsverlaufs, die oft mit einer Verlängerungen der Reaktionszeit einhergehe (vgl. Ebd.). Insgesamt bevorzugen Hirschfeld et al. (Ebd.: 45) den Terminus Kommunikationskonflikt vor allem für phonetische Formprobleme, die in der gegebenen Kommunikationssituation offenbare Konfrontationen auslösen. Diese sind insbesondere für die interkulturelle Kommunikation kennzeichnend. Bei phonetischen Formproblemen handelt es sich einerseits um eine unangemessene Realisierung der phonologischen bedeutungsunterscheidenenden Lautmerkmale. Andererseits können ebenso Unangemessenheiten im suprasegmentalen Bereich zu Störungen bzw. Konflikten führen, da diese - so Veličkova (2004: 4) - nicht nur „die Wahrnehmung von kommunikativen Typen von Äußerungen stören“ (Ebd.). Vielmehr verursachen sie unerwartete, unadäquate emotionale Reaktionen, indem ,[n]och vor dem Erfassen der lexikalischsemantischen Einheiten oder parallel dazu [...] der emotionale Charakter der Rede wahrgenommen" (Ebd.) wird: Die Wahrnehmung der gesprochenen Sprache habe somit eine emotionale Basis und geschehe durch emotionale Grundkategori- 
en (Ebd.). Laut Veličkova (Ebd.) kann dabei der expressive Ausdruck von emotionalen Zuständen in zwei Sprachen nicht zusammenfallen, was bereits am Anfang der sprechsprachlichen Kommunikation eine Basis für kommunikative Störungen schafft und somit für die Wahrnehmung des Gesprochenen ,manchmal ungeachtet der adäquat wahrgenommenen semantischen Information der gesprochenen Äußerungen“ (Ebd.) bestimmend ist (zu Emotionen als Störungsquelle s. auch weiter unten). In dem vorliegenden Beitrag wird allerdings davon ausgegangen, dass im internationalen Sprachkontakt nicht nur phonetische, sondern auch andere formbedingte Abweichungen, wie etwa auf der Ebene der Lexik und Syntax, zur Beeinträchtigung des kommunikativen Ablaufs führen und somit eine schwerwiegende Ursache für konkrete Konfrontationen sein können. Für die weiteren Ausführungen kommen folglich beide Begriffe - der Störungs- und der Konfliktbegriff - gleichwertig in Betracht. Jedes Mal treten nämlich möglicherweise Verstehens- und Verständigungsprobleme auf, die es unmöglich machen, die geplante Kommunikationsabsicht zu realisieren.

Demgegenüber entstehen kulturbedingte Kommunikationsstörungen bzw. -konflikte in verschiedenen weiteren Bereichen, in denen u.a. mittels Sprache als beschreibbares Zeichensystem intentional-sozial gehandelt wird, und nicht infolge sprachlicher Interferenzerscheinungen. Kulturbedingte Kommunikationsstörungen bzw. -konflikte sind, wie einleitend erwähnt, in kulturellen Überschneidungssituationen verortet. D.h., sie kommen dann zustande, wenn unterschiedliche Kulturen als Zeichen-, Wissens- und Orientierungssysteme in ihrer Mehrdimensionalität aufeinanderstoßen (s. auch Kap. 1). Im Allgemeinen liegt mit kulturbedingten Kommunikationsstörungen bzw. -konflikten ein breites Spektrum an kommunikativen Erscheinungen vor, mit denen die Kommunikationsinteragierenden jeweils konfrontiert werden. Ein Beispiel dafür kann die Kulturspezifik von Texten / Textsorten genannt werden. Im Hinblick auf die universitäre Lehr-Lern-Kommunikation macht Venohr (2008: 310) diesbezüglich auf zwei wesentliche Aspekte aufmerksam - zum einen auf die mögliche Null-Äquivalenz der jeweiligen Textsorte in der Ausgangskultur, zum anderen auf deren partielle Äquivalenz, die sich folgendermaßen manifestiert: (1) in sekundären Textfunktionen (z.B. Orientierung auf den Erklärungsgegenstand vs. stärkere Handlungsorientierung in japanischen Vorträgen im Vergleich zu deutschen); (2) in der Interaktionsmodalität (ernst vs. unernst in mündlichen Prüfungen) (3) in textsortenspezifischen Illokutionsprofilen (auf ,Geschichten' bei Anliegensformulierungen in deutschen Sprechstunden verzichten); (4) in intertextuellen Bezügen (z.B. Unterschiede beim Zitieren und Bewerten von Sekundärliteratur) sowie (5) in einzelsprachlichen Realisierung wissenschaftssprachlicher Sprachhandlungen (z.B. bezüglich der Kategorie Direktheit/Indirektheit) (dazu vgl. auch Günthner 2011: 21 ff.).

Eine weitere Ursache für Probleme in der interkulturellen Kommunikation in Mündlichkeit können Unterschiede im Unterbrechungs- und Schweigeverhalten darstellen, die - wie es z.B. in finnisch-deutschen Gesprächen der Fall ist - prin- 
zipiell ein Konfliktpotential enthalten (vgl. Olbertz-Siitonen 2003: 249 ff.). Die jeweiligen Unterbrechungen, die als solche auf Grund konkreter Besonderheiten (deren Merkmale und Mittel, Rederechtbehauptung und Interventionsformen) erkannt werden, rufen dabei konkrete Reaktionen, darunter unterschiedliche kulturbedingte Unterbrechungstoleranz, hervor (vgl. Ebd.: 255 f.). Auf Grund möglicherweise hemmender Wirkung gelten ferner, wie bereits erwähnt, ebenfalls Emotionen als eine durchaus gravierende Störungsquelle, die gerade in der interkulturellen Kommunikation besonders ernst zu nehmen ist (vgl. Stock 2003: 355). Diesbezüglich seien mit Stock (Ebd.: 356) folgende Prämissen hervorgehoben: (1) die Universalität der Emotionen als menschliche Anlage und deren Unlösbarkeit aus individuums- und soziokulturspezifischen Bedeutungszusammenhängen; (2) das wechselseitige Verhältnis zwischen dem Grad des emotionalen Verstehens (als eine richtige situativ angemessene Interpretation von Verhalten) und dem Grad der Ähnlichkeit zwischen den eigenen Erfahrungen und denen der Partner; (3) die individuell geprägten Erfahrungen hinsichtlich der individuellen Kommunikationsbiographie und das damit zusammenhängende interpretativ-deutende Verstehen von Emotionen als Annäherung an die Intentionen des Partners und (4) die soziokulturelle Bedingtheit der Emotionen und die daraus resultierende Schwierigkeit im Hinblick auf deren Kommunikation unter interkulturellen Bedingungen. Aus sprechwissenschaftlicher Sicht soll Stock zufolge insbesondere der Frage nachgegangen werden, ,welche Mittel für die Expression und Perzeption genutzt werden“" (Ebd.: 361). In den Fokus der Untersuchungen sind allerdings nicht nur die prosodischen Merkmale zu stellen, da die Kommunikation von Emotionen über mehrere Kanäle erfolgt und somit auf verschiedenen Ebene - der semantischen, lexikalischen, syntaktischen und lautlichen - gleichzeitig zu verorten ist (vgl. Ebd.: 362). Schließlich kann es auch auf der handlungspragmatischen Ebene zu Störungen / Konflikten kommunikativer Abläufe kommen, indem beispielsweise ein unangemessener Einsatz der Modalverben das Gelingen eines Beratungsgesprächs in universitären Begegnungssituationen verhindern kann (mehr dazu vgl. Venohr 2008: 311).

Abschließend sei mit Liedke (1998: 198) betont, dass Kommunikationsstörungen bzw. -konflikte im fremdsprachlichen Handeln als Normalität gelten. Sie kommen vor allem dadurch zustande, dass ,eine(r) der Kommunikationsbeteiligten die jeweilige Verständigungssprache zumeist nicht ,perfekt" beherrscht" (Ebd.). Die sich aus diesem Umstand ergebenden Verständigungsschwierigkeiten, die den Interaktionspartnern jeweils durchaus bewusst werden, lassen den nativ-nonnativen Diskurs „als Diskurs ausdrucksmäßig reduzierter Persönlichkeiten“ (Kotthoff 1991: 394) gelten. So kann z.B. die Einschränkung im Bereich abtönender und verstärkender Redemittel, wie etwa in deutsch-anglo-amerikanischen Gesprächen, dazu führen, dass ,deren Bedeutung nicht angemessen erlebt wird“ (Ebd.). Neben sprachlichen Interferenzen bzw. dem aus sprachlichen Defiziten resultierenden sog. „foreigner talk“ als vereinfachtes Sprachverhalten der nonnativen Sprecher/-innen gegenüber 
der Zielsprache gelten also, wie bis jetzt erläutert, als mögliche Ursachen für Kommunikationsstörungen / -konflikte zusammenfassend: textsortenspezifische Aspekte, kulturbedingtes pragmatisch-kommunikatives Verhalten der Interaktionsbeteiligten, das sich in der spezifischen Realisierung konkreter Handlungsmuster manifestiert wie auch der emotionale Ausdruck. Hinzu kommen ferner unterschiedliche Wissensbestände der Kommunikationspartner, die sich ebenfalls aus ihrer Zugehörigkeit zu verschiedenen Kulturen im weiteren Sinn ergeben (dazu vgl. auch Knapp / Knapp-Potthoff 1990: 62 ff.). Zu nennen ist an dieser Stelle letztlich ebenfalls der erwartungswidrige bzw. situativ unangemessene Einsatz nonverbaler Komponenten in den multimodal ausgerichteten sprechsprachlicher Äußerungen.

\section{Empirische Studie}

\subsection{Das Untersuchungsdesign}

Die durchgeführte Studie stellt lediglich einen Ausschnitt des größeren Forschungsvorhabens zur Wirkung, Verständlichkeit und zur rhetorischen Kompetenz polnischer Deutschlernender in der universitären Wissenschaftskommunikation dar. Das Ziel der Studie war, wie einleitend formuliert, der Problematik möglicher Kommunikationsstörungen - deren Ursachen sowie Konsequenzen - nachzugehen, die bei der universitären Wissensvermittlung im interkulturellen Kontext auftreten können.

Das Testmaterial für die vorliegende Studie umfasste zwei Aufnahmen von studentischen Seminarreferaten im Deutschen als Fremdsprache, von denen jeweils die ersten vier Minuten einer Analyse unterzogen wurden. Die Sprecherinnen, zwei polnische Germanistikstudentinnen der Universität Łódź, referierten in einem fachbezogenen Seminar zu Inhalten aus dem Bereich der Didaktik und Methodik des fremdsprachlichen Unterrichts. Die Themen der Referate lauten entsprechend: „Lehrperson unter besonderer Berücksichtigung der wichtigsten Berufskompetenzen in Bezug auf den Fremdsprachenunterricht" (Sprecherin 1) und „Gruppenarbeit im Fremdsprachenunterricht“ (Sprecherin 2). Zum Zeitpunkt der Aufnahme studierten beide Sprecherinnen im 6. Semester / BA, ihr Sprachniveau im Deutschen war - zumindest curricular vorausgesetzt $-\mathrm{C} 1$, sie verfügten über Englischkenntnisse und waren 22 Jahre.

Die Einbettung der vorliegenden Studie in eine interkulturell geprägte Wissenschaftskommunikation mit Fokus auf das studentische Seminarreferat setzte eine entsprechende Probandenauswahl voraus, die vorrangig durch folgende Kriterien bestimmt wurde: (1) die Ausgangs-/Erstsprache - es wurden Proband/-innen mit Deutsch als Mutter- bzw. Erstsprache gewählt (die von Nichtmuttersprachler/-innen gewonnenen Daten wurden bei der Auswertung nicht berücksichtigt); (2) den sozialen Status - die Proband/-innen sollten, ebenso wie die Sprecher/-innen, einen uni- 
versitären Status haben (zur Zeit der Analyse sollten sie an einer deutschen Universität immatrikuliert sein); (3) den authentischen Forschungskontext - die audiovisuelle Analyse des Testmaterials sollte im Rahmen von Lehrveranstaltungen an einer deutschen Universität erfolgen, sodass eine annähernd authentische Kommunikationssituation geschaffen wird, in der polnische Referent/-innen als potenzielle ErasmusStudent/-innen ein Referat vor überwiegend deutschsprachigen Kommiliton/-innen in der Zielsprache Deutsch halten; (4) den thematischen Inhalt des Testmaterials aus dem Bereich der Geisteswissenschaften (Sprachwissenschaft / Didaktik und Methodik des Fremdsprachenunterrichts etc.) - die Proband/-innen sollten mindestens ein geisteswissenschaftliches Fach studieren bzw. studiert haben, um eine allgemeine Vorstellung von den im Referat übermittelten Problemfeldern zu haben.

An der durchgeführten Untersuchungsrunde nahmen 71 deutschsprachige Studierende aus dem Seminar für Sprechwissenschaft und Phonetik der MartinLuther-Universität in Halle-Wittenberg teil - 32 davon analysierten die Aufnahme 1 und 39 - die Aufnahme 2. Der Versuch erfolgte nach einer standarisierten Aufgabenstellung und beruhte auf einer audiovisuellen, qualitatv-quantitativen Analyse der aufgenommenen Referate. Die Proband/-innen durften sich das Testmaterial nur einmal anhören und -sehen. Zu dem vorliegenden Versuchsteil gehörten zwei Fragen, die einen Ausschnitt eines umfangreicheren Fragebogens ausmachten Frage 1: Was hat Sie gestört, dem Inhalt des Referats zu folgen? Nennen Sie bitte mögliche Störungsfaktoren; Frage 2 - Wie anstrengend war es für Sie, der Referentin zuzuhören? Bei der ersten Frage handelt es sich um eine klassisch offene Frage, bei der die Hörer/-innen jeweils beliebig viele Faktoren aufzählen durften. Bei der zweiten Frage wurde den Proband/-innen ein Polaritätsprofil in Form einer 6-Stufen-Skala zur Einschätzung der Referate hinsichtlich des Anstrengungsgrades beim Zuhören / -sehen vorgelegt (eine geschlossene Frage); anschließend wurden die Testpersonen gebeten, das jeweilige Hörurteil mit eigenen Anmerkungen zu begründen (eine offene Frage). Im Hinblick auf die aktuelle Zielsetzung mag die zweite Frage nur auf den ersten Blick ungeeignet sein. Dabei wurde hier von der Annahme ausgegangen, dass der jegliche, subjektiv empfundene (zu) hohe, Anstrengungsgrad (bzw. Konzentrationsaufwand) beim Zuhören / -schauen ebenfalls als wesentlicher Störungsfaktor in der gegebenen Redesituation zu betrachten ist, für den konkrete Ursachen verantwortlich sein können.

\subsection{Die Darstellung der Untersuchungsergebnisse}

Im Folgenden sollen die wichtigsten, aus dem aktuellen Versuchsteil gewonnenen Ergebnisse zusammenfassend vorgestellt werden. Die Übersicht 1 und Übersicht 2 verschaffen zunächst einen Überblick über mögliche Faktoren, die die deutschsprachigen Hörer/-innen gestört haben, dem Inhalt des jeweiligen Referats im Deutschen als Fremdsprache zu folgen (Frage 1). Zur Orientierung über 
das mögliche Ausmaß der jeweiligen Störungsursache wird zusätzlich die Anzahl der jeweils von Proband/-innen abgegebenen Nennungen bezüglich der einzelnen Faktoren bzw. Faktorenbereiche aufgeführt.

Übersicht 1. Die empirisch aufgedeckten Störungsfaktoren beim Verfolgen des Referats - Sprecherin 1

\begin{tabular}{|l|l|}
\hline Sprechweise & $\begin{array}{l}\text { (zu schnelles) Sprechtempo, fremder Akzent, fehlende Lösungstie- } \\
\text { fe, kaum Pausen, Häsitationen; Betonungsabweichungen; artikula- } \\
\text { torische Unangemessenheiten, (zu hohe) Sprechspannung-70 Nen- } \\
\text { nungen }\end{array}$ \\
\hline Sprachliche Mittel & $\begin{array}{l}\text { komplexer Satzbau, darunter zahlreiche Schachtelsätze, unange- } \\
\text { messene Wortwahl, Nominalstil, keine freie Rede (abgelesener Vor- } \\
\text { trag) - 4 Nennungen }\end{array}$ \\
\hline Körperausdruck & $\begin{array}{l}\text { kaum Blickkontakt, unangemessene Gestik (auffallendes Umblät- } \\
\text { tern der zu großen Notizzettel), unangemessenes Raumverhalten (zu } \\
\text { häufige Zuwendung zum Laptop) - 4 Nennungen }\end{array}$ \\
\hline $\begin{array}{l}\text { Gliederung und / oder Auf- } \\
\text { bau ggf. Inhalt und Argu- } \\
\text { mentation }\end{array}$ & $\begin{array}{l}\text { inhaltliche Wiederholungen, schwer erkennbarer roter Faden (ge- } \\
\text { hemmter Sprechdenkprozess), Fülle an Informationen - 4 Nen- } \\
\text { nungen }\end{array}$ \\
\hline Hörerorientierung & $\begin{array}{l}\text { unpersönliches Vorgetragen, fehlende Ansprechhaltung, hörerun- } \\
\text { freundliche ,Verpackung des Inhalts”- 3 Nennungen }\end{array}$ \\
\hline
\end{tabular}

Übersicht 2. Die empirisch aufgedeckten Störungsfaktoren beim Verfolgen des Referats - Sprecherin 2

\begin{tabular}{|l|l|}
\hline Sprechweise & $\begin{array}{l}\text { artikulatorische Abweichungen, Häsitationen, (zu schnelles) Sprecht- } \\
\text { empo, fremder Akzent, monotone Melodieführung, kaum Pausen } \\
\text { im Redefluss, fehlende Lösungstiefe, Betonungsabweichungen, } \\
\text { (zu) hohe und (zu) enge Stimme, Staccato-Rhythmus / abgehackte } \\
\text { Sprechweise); (zu) hohe Sprechspannung, ,verwaschene" (undeut- } \\
\text { liche) Sprechweise - 69 Nennungen }\end{array}$ \\
\hline $\begin{array}{l}\text { Sprache } \\
\text { (sprachliche Mittel) }\end{array}$ & $\begin{array}{l}\text { Satzbauschwierigkeiten (grammatikalische Abweichungen), stel- } \\
\text { lenweise Wortfindungsschwierigkeiten bzw. unangemessene Lexik, } \\
\text { (z.T. unverständlich) - 15 Nennungen }\end{array}$ \\
\hline $\begin{array}{l}\text { Gliederung und / oder Auf- } \\
\text { bau ggf. Inhalt und Argu- } \\
\text { mentation }\end{array}$ & $\begin{array}{l}\text { unangemessene Beispiele und Begriffserklärungen, Defizite in Glie- } \\
\text { derung und damit zusammenhängende inhaltliche Wiederholungen, } \\
\text { schwer erkennbarer roter Faden - 7 Nennungen }\end{array}$ \\
\hline Körperausdruck & $\begin{array}{l}\text { Blick zur Seite - auf den Laptop (nur beim Ablesen), kaum Blick- } \\
\text { kontakt - 1 Nennung }\end{array}$ \\
\hline $\begin{array}{l}\text { Hörerorientierung } \\
\text { (bzw. Sonstiges) }\end{array}$ & $\begin{array}{l}\text { auffällige unvorbereitete Passagen fürs Vortragen, Geräusche im } \\
\text { Raum, eine (audiovisuelle) Aufnahme statt einer echten face-to-fa- } \\
\text { ce-Kommunikation) - 3 Nennungen }\end{array}$ \\
\hline
\end{tabular}


Die in der aktuellen Untersuchungsrunde ermittelten potenziellen Ursachen für Störungen beim Verfolgen der Referate ließen sich beim näheren Betrachten einigen konkreten Kategorien zuordnen. Im Einzelnen kommt es, wie es den beiden Übersichten zu entnehmen ist, in der Reihenfolge der Auftretenshäufigkeit der jeweiligen Störungen auf folgende Kategorien an: die Sprechweise (paraverbale Ebene), die Sprache (sprachliche Mittel, Sprachebene), den Körperausdruck (nonverbale Ebene), die Gliederung und / oder den Aufbau des Referats ggf. den Inhalt und die Argumentation (denklogische Ebene) sowie nicht zuletzt die Kategorie der Hörerorientierung.

Auf Grund der Eintragungen auf der 6-Stufen-Skala zur Frage nach dem Anstrengungsgrad (sprich: dem Konzentrationsaufwand) auf Seiten der Proband/innen beim Zuhören (Verfolgen der Referate) (Frage 2) wurde jeweils ein Medianwert errechnet, der für beide Sprecherinnen 2 betrug - bei , 1 ' für ,sehr anstrengend und ,6“ für ,gar nicht anstrengend'. Die einzelnen Markierungen auf der Skala verteilten sich folgendermaßen (s. die Übersicht 3):

Übersicht 3. Zur Frage nach dem Konzentrationsaufwand auf Seiten der Hörer/-innen - Anzahl der Markierungen auf der 6-Stufen-Skala

\begin{tabular}{|c|c|}
\hline Sprecherin 1 (bei 32 Hörer/-innen) & Sprecherin 2 (bei 39 Hörer/-innen) \\
\hline $\begin{array}{l}1 \text { - } 8 \text { Markierungen; } 2 \text { - } 14 \text { Markierungen; } \\
3 \text { - } 4 \text { Markierungen, } 4 \text { - } 5 \text { Markierungen und } \\
5 \text { - eine Markierung }\end{array}$ & $\begin{array}{l}\text { 1-6 Markierungen, } 2-16 \text { Markierungen, } \\
3-11 \text { Markierungen, } 4-2 \text { Markierungen, } \\
\text { 5-4 Markierungen }\end{array}$ \\
\hline
\end{tabular}

Die Ursachen für das anstrengende Zuhören /-schauen und somit für das erschwerte Verfolgen der Referate, die aus den Begründungen der Hörurteile ermittelt werden konnten, ließen sich ebenfalls den bereits oben verwendeten Kategorien zuordnen. Die Angaben in der folgenden Übersicht wurden hier ebenfalls um die Anzahl der probandenseitigen Nennungen bezüglich möglicher Ursachen ergänzt (s. Übersicht 4).

Übersicht 4. Mögliche Ursachen für erschwertes Verfolgen der Referate (anstrengendes Zuhören/ -sehen)

\begin{tabular}{|c|c|}
\hline Sprecherin 1 & Sprecherin 2 \\
\hline $\begin{array}{l}\text { Sprechweise: } \\
\text { fremder Akzent, zu schnelles Sprechtempo, feh- } \\
\text { lende Lösungstiefe, Abweichungen im Rhyth- } \\
\text { mus, abweichende Melodieführung, Betonungs- } \\
\text { abweichungen, unangemessene Sinneinheiten, } \\
\text { kaum Pausen, Häsitationen (ähs) - } 40 \text { Nen- } \\
\text { nungen }\end{array}$ & $\begin{array}{l}\text { Sprechweise: } \\
\text { Häsitationen (,ähs"), segmentale Abweichungen, } \\
\text { unangemessenes Sprechtempo, fehlende Lö- } \\
\text { sungstiefe, unangemessene Melodieführung, } \\
\text { Abweichungen im Rhythmus, Betonungsabwei- } \\
\text { chungen, Schmatzgeräusche/-laute - } 31 \text { Nen- } \\
\text { nungen }\end{array}$ \\
\hline
\end{tabular}




\begin{tabular}{|l|l|}
\hline $\begin{array}{l}\text { Körperausdruck: } \\
\text { störende Gestik, unzureichender Blickkontakt, } \\
\text { lästiges Umblättern der Notizzettel, Unange- } \\
\text { messenheiten hinsichtlich der PowerPointPrä- } \\
\text { sentation - 5 Nennungen }\end{array}$ & $\begin{array}{l}\text { Sprachliche Mittel: } \\
\text { unangemessene Wortwahl, Suchen nach entspre- } \\
\text { chendem Wortschatz, grammatikalische Abwei- } \\
\text { chungen bezüglich des Satzbau - 11 Nennungen }\end{array}$ \\
\hline $\begin{array}{l}\text { Gliederung und / oder Aufbau, ggf. Inhalt } \\
\text { und Argumentation } \\
\text { unangemessene Gliederung, keine Beispiele, } \\
\text { unklares Thema (trivialer Inhalt), kaum Erläu- } \\
\text { terungen zum Inhalt - 4 Nennungen }\end{array}$ & $\begin{array}{l}\text { Gliederung und / oder Aufbau, ggf. Inhalt } \\
\text { und Argumentation } \\
\text { unklare Beispiele, fehlende Gliederung, inhalt- } \\
\text { liche Wiederholungen - 6 Nennungen }\end{array}$ \\
\hline $\begin{array}{l}\text { Sprachliche Mittel: komplexer Satzbau - } \\
1 \text { Nennung }\end{array}$ & $\begin{array}{l}\text { Hörereigene Dispositionen: hörereigene } \\
\text { Schwierigkeiten - 4 Nennungen }\end{array}$ \\
\hline $\begin{array}{l}\text { Hörereigene Dispositionen: hörereigene Schwie- } \\
\text { rigkeiten - } 8 \text { Nennungen }\end{array}$ & $\begin{array}{l}\text { Hörerorientierung: fehlende Ansprechhal- } \\
\text { tung, unangemessene PowerPointPräsentation } \\
-3 \text { Nennungen }\end{array}$ \\
\hline $\begin{array}{l}\text { Hörerorientierung: fehlende Ansprechhaltung } \\
-1 \text { Nennung }\end{array}$ & Körperausdruck: keine Angaben \\
\hline
\end{tabular}

Mit der in der Übersicht 4 zusätzlich angeführten Kategorie der hörereigenen Dispositionen sind im Allgemeinen hörereigene (subjektive) Schwierigkeiten, dem Inhalt zu folgen, wie etwa momentane Müdigkeit bzw. unzureichende Konzentrationsfähigkeit, gemeint.

\subsection{Die Zusammenfassung und die Diskussion der Ergebnisse}

Die in der vorliegenden Untersuchungsrunde aufgedeckten Ursachen für erschwertes Verfolgen der Referate bzw. für den recht erhöhten Anstrengungsgrad beim Zuhören /-schauen (der Median 2 beim Wert , 1' für ,sehr anstrengend', s.o.) sind allgemein breit gefächert. Sie liegen, wie in den obigen Übersichten gezeigt werden konnte (s. Kap. 5.2), in unterschiedlichen Bereichen, wobei zunächst der Bereich der Sprecherperson (situativ bedingte/-er Sprechweise Körperausdruck) und der der Äußerung (deren segmentale und suprasegmentale Realisierung, deren Gliederung und / oder Aufbau, ggf. Inhalt und Argumentation) zu nennen sind. Zugleich treten sie offenbar in verschiedenen Kombinationen auf, die sich u.a. in der unzureichenden Hörerorientierung beim Gestalten des Referats bzw. beim Vortragen selbst manifestieren, auch wenn diese Kategorie als solche von den Proband/-innen lediglich einige Male genannt wurde. Die Kombination verschiedener Kategorien, in denen potenzielle Ursachen für Kommunikationsstörungen / -konflikte angesiedelt sind, resultiert dabei aus der in Kapitel 2 erläuterten Multimodalität kommunikativer Vorgänge in Mündlichkeit.

Im Allgemeinen gelten als potenzielle Ursachen für Störungen in der untersuchten Kommunikationssituation sowohl bei Sprecherin 1 als auch Sprecherin 2 vor allem paraverbale Komponenten - in den beiden Fällen waren es die meisten 
Nennungen, die von Proband/-innen abgegeben wurden (70 und 69). Gemeint sind damit, wie oben aufgeführt, u.a. Unangemessenheiten im Bereich der Melodieführung, Betonung und Artikulation, die gleichzeitig zum großen Teil interferenzbedingt sind und deshalb unter dem Begriff des fremden Akzents zusammenzufassen sind. Ihrerseits können sie insbesondere unzureichender Verständlichkeit der übertragenen Inhalte zugrunde liegen oder sogar im schlimmsten Fall zum Abbruch des Kommunikationsgeschehens führen, indem der Rezipient nicht (mehr) im Stande ist, die perzipierten Signale inhaltlich aufzunehmen. Weitere Unangemessenheiten bezüglich der paraverbalen Aspekte betreffen die untersuchte Redesituation, in der es den Kommunikationspartnern als Rezipienten ermöglicht werden sollte, den mündlich übermittelten Informationen möglichst problemlos folgen zu können und diese möglichst weitgehend zu behalten, damit die kommunikative Funktion des Referats (Informieren) erfüllt werden könnte. Dies wäre z.B. durch fließendes Sprechen ohne (auffallende) Häsitationen, situativ angemessene, keinesfalls monotone Melodieführung und Betonung bzw. eine nicht zu hohe Sprechspannung o.ä. zu sichern, sodass in dem Referat statt Nervosität und Unruhe bzw. Unsicherheit der Sprecherperson deren Glaubwürdigkeit, Fachkompetenz sowie Engagement vermittelt werden kann.

Als störend beim Verfolgen der Referate erwiesen sich darüber hinaus auch Faktoren, die außerhalb des Bereichs liegen und die des Öfteren nicht vorab vorauszusehen sind, wie etwa zusätzliche (Außen-)Geräusche bzw. sogar Lärm (von der Straße oder vom Nebenraum). Ferner war es die Kommunikationssituation selbst, in der sich die Proband/-innen befanden: Sie sollten die Referate auf Grund audiovisueller Aufnahmen beurteilen und wurden dadurch mit einem gewissen Grad an Unvertrautheit bezüglich der Situation konfrontiert, während die Rezeption studentischer Referate in der Regel in einer face-to-face-Kommunikation stattfindet. Wenn man aber bedenkt, dass zahlreiche technische Einrichtungen zur Übertragung von Ton und Bild auch in der universitären Wissenschaftskommunikation der Gegenwart keine Neuigkeit mehr sind, ist solche - immer noch direkte Kommunikationssituation, wie etwa bei einer Video-Konferenz durchaus möglich. Betrifft sie vielleicht noch lange nicht das Vortragen in Form des studentischen Seminarreferats, wird sie jedoch mittlerweile bei Dissertationsverteidigungen auf der internationalen Ebene praktiziert.

Schließlich seien auch hörerseitige Dispositionen als Ursache für Störungen der kommunikativen Abläufe notwendigerweise berücksichtigt. Diese können nämlich durch die oben aufgeführten Problemfelder der Kommunikation (vgl. Übersichten 1, 2 und 4 - Kap. 5.2), die in ihrer Komplexität in unzureichender Hörerorientierung der Sprecherperson Ausdruck finden, weitgehend abgeschwächt werden. Die Zuhörer/-innen, die einer konkreten Redesituation, wie etwa dem studentischen Seminarreferat, jeweils mit eigenen kulturell bzw. individuell bedingten Erwartungen entgegen kommen, können auf Grund der hier aufgedeckten Störungsfaktoren auf eine aktive Teilnahme an der gegebenen Kommunikati- 
onssituation verzichten. Folglich wird die von der Sprecherperson beabsichtigte Kommunikationswirkung schlechthin nicht erzielt, sodass sich die Rezipienten ungenügend informiert fühlen.

\section{Abschließende Bemerkungen}

Das Vortragen stellt nicht nur eins der wesentlichsten Instrumente zum Austausch wissenschaftlicher Erkenntnisse unter Wissenschaftlern dar. Mit Redesituationen werden im universitären Bereich ebenfalls Studierende konfrontiert, die vor einer Seminargruppe (und vor einem Dozenten / einer Dozentin) ein Referat halten sollen, um das im Selbststudium erworbene Wissen an andere Studierende zu vermitteln und zugleich mit dieser Leistung, wie das etwa im deutschsprachigen Raum der Fall ist, einen Qualifikationsnachweis zu erwerben. In der interkulturellen Kommunikation ist dabei, wie an den hier untersuchten Beispielen gezeigt werden konnte, mit konkreten Schwierigkeiten zu rechnen, die die universitäre Wissensvermittlung sogar weitgehend stören können. Das ist mit Sicherheit einer der Gründe, warum im ausländischen Germanistikstudium, z.B. in Polen, auf das studentische Seminarreferat als Wissensvermittlungs- bzw. Leistungsmessungsform des Öfteren verzichtet wird. Damit ist jedoch das Problem nicht gelöst. Viele polnische Germanistikstudierende werden nämlich im Rahmen verschiedener internationaler Bildungsprogramme, wie z.B. Erasmus, , potenziell vor die Aufgabe gestellt, in der Zielsprache Deutsch vortragen zu müssen. Statt also das Vortragen als universitäre Arbeitsform zu vermeiden, sollten vielmehr potenzielle Faktoren als Ursachen für Kommunikationsstörungen / -konflikte jeweils vor Augen geführt werden. Das setzte sich der vorliegende Beitrag zum Ziel.

Den potenziellen Störungsfaktoren, die z.T. in der hier dargestellten Untersuchungsrunde aufgedeckt wurden, ist bereits in den Prozessen des fremdsprachlichen Lehrens und Lernens bestenfalls vorab ständig zu reflektieren, was u.a. in zweckgerichteten Redetrainings weitgehend vollzogen werden kann. Somit kann der jegliche Anstrengungsgrad auf der Seite des Rezipienten vermindert und somit der durch ihn aufzubringende Konzentrationsaufwand reduziert werden. Die bewusste Reflexion soll jeweils auf Grund empirischer Untersuchungen im Hinblick auf konkrete Kommunikationssituation erfolgen, wie das am Beispiel einer Redesituation in der interkulturellen Lehr-Lern-Kommunikation gezeigt wurde. Im Fokus interkulturell ausgerichteter Redetrainings sollen zusammenfassend insbesondere folgende Aspekte stehen: die Arbeit an möglichen systeminhärenten, z.T. interferenzbedingten Spracherscheinungen (Lexik, Grammatik, Phonetik) einerseits, andererseits die Arbeit an situativer Angemessenheit, die sich auf verschiedenen Ebenen (der verbalen, para- und nonverbalen) gleichzeitig abspielt und sich in der Hörerorientierung manifestiert. Nur damit kann die kommunikative 
Funktion des studentischen Seminarreferats erfüllt werden, die im universitären Unterrichtsalltag an dem Grad des Behaltens der durch die Sprecherperson vermittelten Wissensbestände grundsätzlich messbar ist.

\section{Literatur}

Deppermann, Arnulf / Spranz-Fogasy, Thomas (2002): Zur Entstehung von Interaktionsstörungen durch zeitliche Kompensierung. In: Verständigungsprobleme und gestörte Kommunikation. Radolfzell. S. 44-62.

Ehlich, Konrad (1993): Deutsch als fremde Wissenschaftssprache. In: Jahrbuch DaF 19, S. 13-42.

Fiehler, Reinhard et al. (2004): Eigenschaften gesprochener Sprache. (Studien zur Deutschen Sprache. Forschungen des Instituts für Deutsche Sprache. Bd. 30). Tübingen.

Grzeszczakowska-Pawlikowska, Beata (2016): Hörerorientierung in der rhetorischen Redepraxis bei polnischen Germanistikstudierenden. In: Hirschfeld, Ursula / Lange, Friderike / Stock, Eberhard (Hrsg.): Phonetische und rhetorische Aspekte in der interkulturellen Kommunikation. (SSP 7). Berlin. S. 77-84.

Guckelsberger, Susanne (2005): Mündliche Referate in universitären Lehrveranstaltungen. Diskursanalytische Untersuchungen im Hinblick auf eine wissenschaftsbezogene Qualifizierung von Studierenden. München.

Günthner, Susanne (2001): Kulturelle Unterschiede in der Aktualisierung kommunikativer Gattungen. In: Info DaF 2001/1, S. 15-32.

Gutenberg, Norbert (2001): Mündlich realisierte schriftkonstituierte Textsorten. In: Brinker, Klaus et al. (Hrsg.): Text- und Gesprächslinguistik. Linguistics of Text and Conversation. Ein internationales Handbuch zeitgenössischer Forschung. Berlin. S. 574-587.

Hausendorf, Heiko (1997): Die Körperlichkeit des Sprechens: Ein Stilmittel der mündlichen Kommunikation? In: Selting, Margaret / Sandig, Barbara (Hrsg.): Sprech- und Gesprächsstile. Berlin/New York. S. 400-426.

Hirschfeld, Ursula / Neuber, Baldur / Stock, Eberhard (2010): Phonetische Sprechwirkungsforschung im Bereich der interkulturellen Kommunikation. In: Hirschfeld, Ursula / Stock, Eberhardt (Hrsg.): Sprechwissenschaftlich-phonetische Untersuchungen zur interkulturellen Kommunikation Russisch-Deutsch. (HSSP 33). Frankfurt am Main. S. 43-67.

Klinger, Jette (2010): Die kulturelle Prägung des mündlichen Seminarreferats. In: Skiba, Dirk (Hrsg.): Textmuster: schulisch-universitär-kulturkontrastiv. Beiträge zur Theorie und Empirie lernersprachlicher Textproduktion. Frankfurt am Main/Berlin/Bern. S. 11-28.

Knapp, Kalfried / Knapp-Potthoff, Annelie (1990): Interkulturelle Kommunikation. In: Zeitschrift für Fremdsprachenforschung 1, S. 62-93.

Kotthoff, Helga (1991): Lernersprachliche und interkulturelle Ursachen für kommunikative Irritationen. Zugeständnisse und Dissens in deutschen, anglo-amerikanischen und nativ-nichtnativen Gesprächen. In: Linguistische Berichte. Forschung Information Diskussion, Heft 133, S. 375-397.

Liedke, Martina (1998): Fremdsprachliches Handeln: Kommunikationsstörung als Normalität. In: Fiehler, Reinhard (Hrsg.): Verständigungsprobleme und gestörte Kommunikation. Opladen/ Wiesbaden. S. 198-215.

Meyer, Dirk (2013): Rede. In: Bose, Ineset et al. (Hrsg.): Einführung in die Sprechwissenschaft. Phonetik, Rhetorik, Sprechkunst. Tübingen. S. 121-125. 
Olbertz-Siitonen, Margarethe (2003): Unterschiede im Unterbrechungs- und Schweigeverhalten als mögliche Ursachen für Probleme in der Kommunikation zwischen Finnen und Deutschen. In: Anders, Lutz Christian / Hirschfeld, Ursula (Hrsg.): Sprechsprachliche Kommunikation. Probleme, Konflikte, Störungen. (HSSP 12). Frankfurt am Main. S. 249-258.

Potapova, Rodmonga K. (2003): Zur Kategorialität der Wahrnehmung der gesprochenen Fremdsprache. Linguistische, para- und extralinguistische Aspekte. In: Anders, Lutz Christian / Hirschfeld, Ursula (Hrsg.): Sprechsprachliche Kommunikation. Probleme, Konflikte, Störungen. (HSSP 12). Frankfurt am Main. S. 271-280.

Quasthoff, Uta (1996): Mündliche Kommunikation als körperliche Kommunikation: Beobachtung zur direkten Interaktion und zum Fernsehen. In: Biere, Bernd Ulrich / Hoberg, Rudolf (Hrsg.): Mündlichkeit und Schriftlichkeit im Fernsehen. Tübingen. S. 9-28.

Scherer, Klaus R. (1970): Non-verbale Kommunikation. Hamburg.

Scollon, Ron / Scollon, Suzanne (1995): Somatic Communication. How useful is ,Orality for the Characterization of Speech Events and Cultures? In: Quasthoff, Uta (Hrsg.): Aspects of Oral Communication. Berlin/New York. S. 9-29.

Stock, Eberhard (2003): Emotionen als Störungsquelle in der interkulturellen Kommunikation. In: Anders, Lutz Christian / Hirschfeld, Ursula: Sprechsprachliche Kommunikation. Probleme, Konflikte, Störungen. (HSSP 12). Frankfurt am Main. S. 355-364.

Straub, Jürgen (2007a): Kultur. In: Straub, Jürgen / Weidemann, Arne / Weidemann, Doris (Hrsg.): Handbuch interkulturelle Kommunikation und Kompetenz. Grundbegriffe - Theorien-Anwendungsfelder. Stuttgart/Weimar. S. 7-24.

Thielmann, Winfried (2009): Wissenschaftliches Sprechen und Schreiben an deutschen Universitäten. In: Dalmas, Martine / Foschi Albert, Marina / Neuland, Eva (Hrsg.): Wissenschaftliche Textsorten im Germanistikstudium deutsch-italienisch-franzoesisch kontrastiv. Trilaterales Forschungsprojekt in der Villa Vigoni (2007-2008). Villa Vigoni. S. 47-54.

Ungeheuer, Gerold (1969): Vorwort. In: Richter, Helmut / Weidmann, Fred (Hrsg.): Semantisch bedingte Kommunikationskonflikte bei Gleichsprachigen. Forschungsbericht 69-2 des Instituts für Kommunikationsforschung und Forschung der Universität Bonn. S. V-X.

Venohr, Elisabeth (2008): Wissenschaftliches Sprechen an deutschen Hochschulen: Indirekte Sprachhandlungen in verschiedenen Textsorten mündlicher Kommunikation. In: Chlosta, Christoph / Lederer, Gabriela / Krischer, Barbara (Hrsg.): Auf neuen Wegen. Deutsch als Fremdsprache in Forschung und Praxis. Tagungsband der 35. Jahrestagung des Fachverbandes Deutsch als Fremdsprache 2007 in Berlin. Göttingen. S. 305-322.

Veličkova, Ludmila (2004): Psycholinguistische phonetische Basis der Störungen bei der interkulturellen sprechsprachlichen Kommunikation. In: Veličkova, Ludmila / Petročenko, Elena (Hrsg.): Klangsprache im Fremdsprachenunterricht (III). Forschung und Praxis. Woronesh. S. 3-11.

Ventola, Eija (2007): Konferenzvorträge: sprechen englisch muttersprachige Konferenzteilnehmer wirklich anders? In: Auer, Peter / Baßler, Harald (Hrsg.): Reden und Schreiben in der Wissenschaft. Frankfurt am Main/New York. S. 115-132.

Wierlacher, Alois (2003): Interkulturalität. In: Wierlacher, Alois / Bogner, Andrea (Hrsg.): Handbuch interkulturelle Germanistik. Stuttgart/Weimar. S. 257-264.

Wiesmann, Bettina (1999): Mündliche Kommunikation im Studium. Diskursanalysen von Lehrveranstaltungen und Konzeptualisierungen der Sprachqualifizierung ausländischer Studienbewerber. (Studien Deutsch 27). München.

Ylönen, Sabine (1994): Die Bedeutung von Textsortenwissen für die interkulturelle Kommunikation. Kommunikative Unterschiede im Biologiestudium an den Partneruniversitäten Jyvaskala und Bonn. In: FINLANCE. A Finnish Journal of Applied Linguistics, Vol. XIII, S. 89-113. 


\section{Abstracts}

Die interkulturelle Kommunikation gehört mittlerweile gegenwärtig zum Alltag der Hochschulen. Dies trifft u.a. für die universitäre Wissensvermittlung zu. Auf Grund der steigenden Studierenden-Mobilität bilden die interkulturell bunt gemischten Seminare einen festen Bestandteil der universitären Lehre. Der interkulturelle insgesamt durchaus positiv zu wertende Austausch unter Studierenden bedeutet allerdings nicht in allen Fällen eine reibungslose Kommunikation. In der kommunikativen Wirklichkeit ist auch potenziell mit gewissen Störungen zu rechnen. Das Erfassen von Ursachen für diese Störungen stellt einen ersten Schritt für die Optimierung der jeweiligen Kommunikationsabläufe dar. Dieser Problematik widmet sich der vorliegende Beitrag am Beispiel einer interkulturell geprägten Redesituation, in der akademisch gesichertes Wissen vermittelt wird.

Schlüsselwörter: interkulturelle Kommunikation, Wissensvermittlung, Kommunikationsstörungen, Optimierung der Kommunikation, Redesituation

\section{Disruptions to the communication of academic knowledge through spoken language}

Intercultural communication has, for some time now, been a feature of everyday life in higher education institutions. This applies also to the communication of academic knowledge acquired by students during their university studies. Increasing student mobility means that interculturally diverse seminars are now a central feature of university teaching and learning but, although the exchange between students certainly ought to be viewed positively, it does not always lead to smooth communication. In real-life communication there may be potential disruptions. Understanding what causes such disruptions constitutes the first step towards optimizing oral interactions. This paper will explore this issue, using the example of a spoken interaction in which academic knowledge is communicated.

Keywords: intercultural communication, knowledge transfer, communication disturbances, optimization of communication, speech situation

Beata Grzeszczakowska-Pawlikowska

Uniwersytet Łódzki

Instytut Filologii Germańskiej

ul. Pomorska 171/173

90-236 Łódź

Polska

E-Mail: beata.pawlikowska@uni.lodz.pl 